\title{
A traceable time interval measurement with a reduced uncertainty
}

\author{
Gihan Gomah* \\ National Institute for Standards (NIS), Time and Frequency LaboratoryGiza, Egypt
}

Received: 17 February 2015 / Accepted: 1 June 2015

\begin{abstract}
Time interval (TI) is one of the basic quantities. Its measurement is not included only in the activities running in the time and frequency metrology but also included in the measurement of many other quantities that depend on time interval measurement (TIM). This means that improving the accuracy and reducing the measurement uncertainty of TI will reflect also on improving the accuracy and reducing the measurement uncertainty of many other quantities. To do that, we need to decrease or eliminate the effect of the dominant factors that degrade the quality of TIM. One of the major contributors to the measurement uncertainty of TI is the internal error of the time interval counter (TIC) used in the measurement. In this paper we introduce a methodology with which we can estimate precisely the internal error of the counter and eliminate its effect.
\end{abstract}

Keywords: Time interval measurement, frequency, time interval counter, uncertainty measurement

\section{Introduction}

Measuring the TI with a reduced uncertainty has a special importance in the field of time and frequency metrology. Building time scales, the calibration of the time transfer systems, and cable delay measurement are essential activities that are running continuously and routinely in the time and frequency laboratories. These activities depend mainly on TIM. In 2003, the Technical Committee of the Time and Frequency (TC-TF) of the European Association of National Metrology (EURAMET) coordinated an inter-laboratory comparison (TF.TI-K1) in order to support the Calibration Measurement Capabilities (CMC) of TIM. In this comparison the delay of a coaxial cable was measured by metrology laboratories of 25 countries as a direct application for TIM. The results of this comparison were not satisfied for the participants due to considerable differences between the participating laboratories [1]. So, EURAMET agreed in 2013 to hold a Time Interval Comparison Pilot Study to have a deeper understanding of the TIM [2].

One of the main likely causes for these differences is the considerable difference between the actual internal error of the counter and the range stated in the counter's manual. Actually, each lab was using the value of the internal error that is stated in the manual. The protocol did not mention a method to measure the actual internal error of the counter before starting the TIM. In the following sections we introduce a methodology with which we can estimate precisely the internal error of the counter in the laboratory.

\footnotetext{
* Correspondence: gihan_gomah@yahoo.com
}

In general, to reduce the uncertainty of TIM all the error sources having a higher contribution to the uncertainty should be studied and minimized. It is known that there are two categories of errors that cause the uncertainty of TIM; the random errors and the systematic errors. Although many of the sources of the random errors can be avoided but their final effect cannot be eliminated at all. On the other hand the sources of the systematic errors cannot be avoided but their effect can be eliminated by calibration. There are three main contributors to the systematic error; the time base error, the trigger level timing error, and the inherent or internal error of the time interval counter (TIC) counter. The effect of both the time base error and the trigger level timing error on degrading the measurement accuracy and uncertainty is insignificant if it was compared to the effect of the internal error of the counter $[3,4]$. The source of the internal error of the counter is the asymmetry in the electronics; which cannot be avoided [5-7]. This type of error in the TIC can degrade significantly the measurement accuracy and uncertainty. So if the value of this internal error is estimated accurately, we can almost eliminate its effect and improve the measurement accuracy and uncertainty.

In this paper, it is proved that the value of the internal error of any TIC is dependent on the type of the TIC, the shape of the signal being measured, the trigger level, and the trigger slope. Also a new technique is proposed for estimating precisely the value of this internal error in the lab. Then, we can almost eliminate the effect of this major contributor to the uncertainty by adding/subtracting its estimated value to/from the measured TI. Finally an example is introduced in order to show a method allowing to improve TI measurements. 


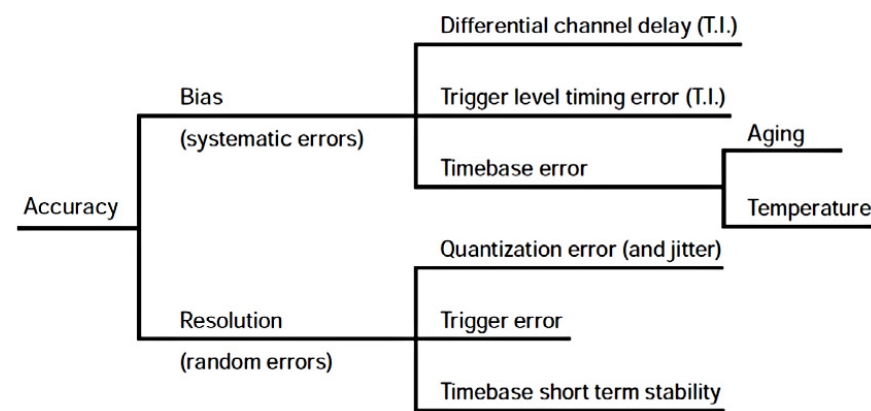

Fig. 1. Sources of errors in TIM $[13,16]$.

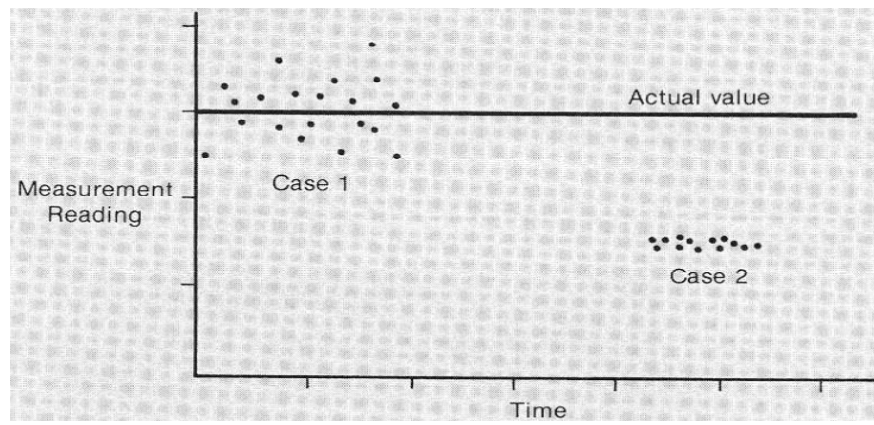

Fig. 2. Case 1 shows the effect of random errors and Case 2 shows the effect of systematic errors in TIM $[13,16]$.

\section{Sources of uncertainty in time interval counters}

Measuring the TI with the least uncertainty is a source of competition between the National Metrology Institutes (NMIs) when they state their CMCs on the Key Comparison Database (KCDB) of the Bureau International des Poids et Mesures (BIPM) [8]. So researchers and manufacturers are continuously searching for new methods and techniques for improving the accuracy and reducing the uncertainty of TIM [9-15]. To reduce the measurement uncertainty all the error sources have to be defined and investigated very well, especially those having the highest contributions. Figure 1 shows the different sources of errors that affect the measurement accuracy and uncertainty of TIM.

Figure 2 shows the effect of the Random errors represented in "Case 1" and the effect of the Systematic Errors represented in "Case 2". The effect of the Systematic Errors on the measurement accuracy are more significant than the Random Errors. At the time the Systematic Errors are identified as the highest contributors that degrade the measurement accuracy and uncertainty; their effect can be cancelled out by calibration. There are three types of systematic errors; the time base error, the trigger level timing error, and the error due to an internal lead/lag in the counter. The error inherent in the counter is called "the internal error of the counter" or simply "the differential channel delay". The contribution of the time base error can be neglected if we used an external time base derived from a highly accurate frequency source (for example, Cesium based frequency source (accurate to parts

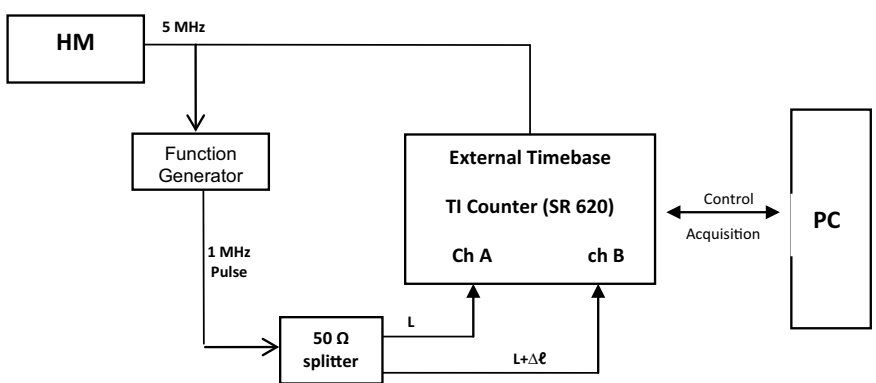

Fig. 3. Schematic for measuring the internal error of a TIC.

in $10^{-13}$ ) or Hydrogen Maser based frequency source (accurate to parts in $\left.10^{-15}\right)$ ). The value of the trigger level timing error is directly proportional to the trigger level and inversely proportional to the slew rate of the signal being measured. So, it is a common practice to use high slew rate signals for TIM to minimize this type of error. On the other hand the error due to the internal error in the counter is an inherent property of the counter that cannot be reduced or controlled. Moreover the value of this internal error may change with time due to the aging of the internal circuitry. Some manufacturers put a ceiling for this internal error for each counte's type. For example, Stanford Research Systems had identified the ceiling of the internal error of their SR620 Time Interval counters to be $\pm 0.5 \mathrm{~ns}$. For the time being, it is a common practice for the users in the time and frequency labs to use this range. Actually, if you are searching for a higher accuracy and less uncertainty you should estimate both the actual value and sign of the internal error. This will be shown in details in the following sections.

\section{Measuring the internal error of the TI}

To estimate the internal error of the TIC, we have to use it in measuring a zero TI. Figure 3 shows a proposed setup to measure a zero TI (with $\Delta \ell=0$ ). In this setup we used a signal having the following specifications: a high slew rate, rise time of $5.1 \mathrm{~ns}, 1 \mathrm{~V}$ peak-to-peak $(0.5 \mathrm{~V}$ to $-0.5 \mathrm{~V}$ ), and pulsed signal of $1 \mathrm{MHz}$. This signal was routed to the two channels of the TIC using a $50 \Omega$ splitter and two cables of equal length. The $1 \mathrm{MHz}$ signal was generated using a function generator its time base was connected to $5 \mathrm{MHz}$ Hydrogen Maser frequency source. A software program was written (using Labview from National Instruments) to control the TIC and acquire the measurement results. All the possible trigger levels were tried/scanned starting from $0.4 \mathrm{~V}$ to $-0.4 \mathrm{~V}$ in a step of $0.05 \mathrm{~V}$. This test was repeated for both the rising and falling edges of the $1 \mathrm{MHz}$ signal. Moreover to test the effect of changing the signal accuracy and the signal shape on the measured error, the same test was repeated twice. The first time using $1 \mathrm{MHz}$ pulsed signal that is based on the internal oscillator of the function generator; which has an accuracy of $2 \times 10^{-6} \mathrm{~Hz} / \mathrm{Hz}$. The second time using a pulse per second (PPS) signal based on UTC (PTB); which is a Hydrogen Maser based signal [17]. To check the 


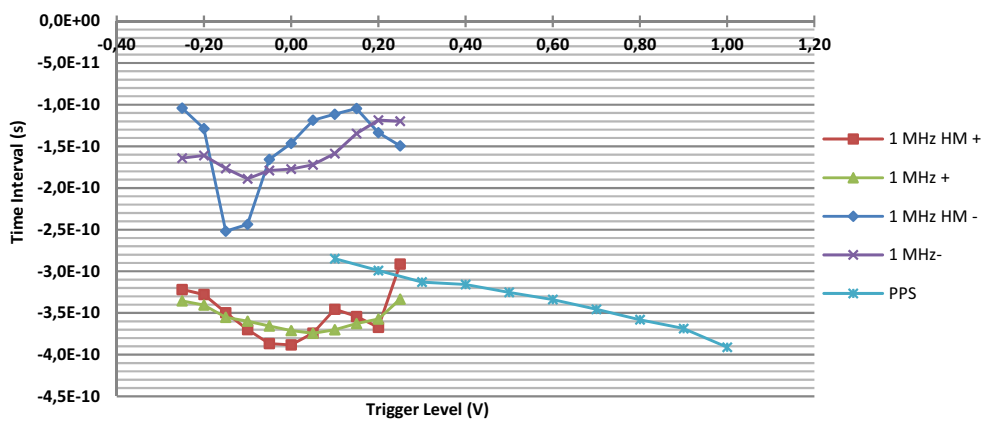

(a): The internal error of TIC\# 1

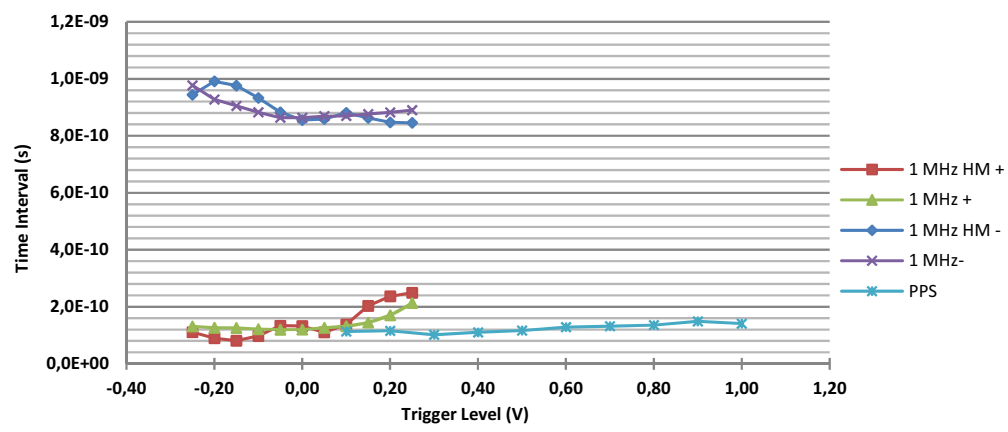

(b): The internal error of TIC\# 2

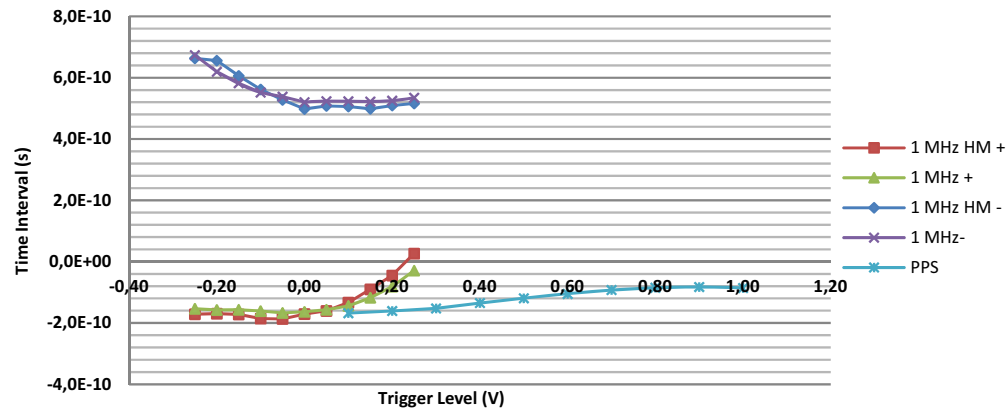

(c): The internal error of TIC\# 3

Fig. 4. The internal error of different TICs using different signals. $1 \mathrm{MHz} \mathrm{HM}+$ : rising edge of $1 \mathrm{MHz} \mathrm{Hydrogen} \mathrm{Maser}$ based signal. $1 \mathrm{MHz}+$ : rising edge of $1 \mathrm{MHz}$ derived from the internal oscillator of the Function Generator. $1 \mathrm{MHz} \mathrm{HM}-$ : falling edge of $1 \mathrm{MHz}$ Hydrogen Maser based signal. $1 \mathrm{MHz}-$ : falling edge of $1 \mathrm{MHz}$ derived from the internal oscillator of the Function Generator. PPS: rising edge of UTC (PTB) (Hydrogen Maser based signal).

reproducibility, all these tests were repeated using three TICs having the same type (SR 620 from Stanford Research Systems). By the way, SR620 TIC is one of the highly accurate TICs in the world and it is used in most of the time and frequency labs in the NMIs. So, many of the investigations on TIM are usually done using this type $[18,19]$. Figure 4 shows the measured internal delay of three different TICs using different signals. It is clear that the internal error of the TIC depends significantly on the signal shape, the triggering slope, and the trigger level. On the other hand, it does not depend significantly on the signal accuracy. Figure 4 a shows that the measured internal error using two signals (the upper two curves $(\times$, $\checkmark)$ ) of the same shape, same triggering slope, and different stability is almost the same. When we changed the triggering slope for the same two signals (the lower two curves
$(\boldsymbol{\Lambda}, \boldsymbol{\square}))$, we get a different value for the internal error. On the other side, when we used a very stable signal with a different shape (PPS), we get a different behaviour for the internal error.

To assure the previous results, in the next section the effect of the asymmetry in the TIC' channels, the $50 \Omega$ splitter' channels, and the cables is investigated.

\section{Investigation of the different sources of asymmetry}

As shown in Figure 5, three different schemes were used to check the effect of the asymmetry that may arises from different sources on the previous results. The previous tests 

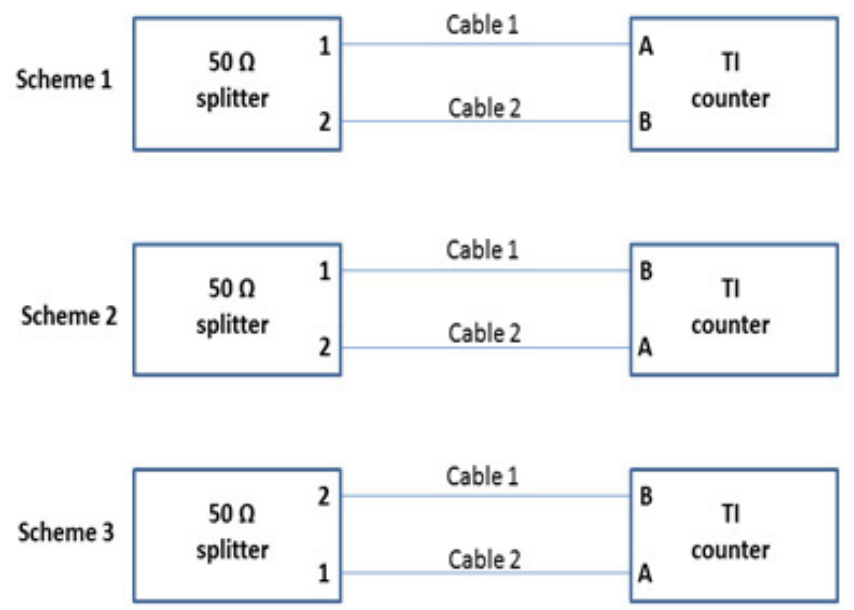

Fig. 5. A schematic shows three schemes for measuring the asymmetry in the TIC' channels, the splitter' channels, and the cables.

were repeated three times using $1 \mathrm{MHz}$ pulsed signal coming from Hydrogen Maser frequency source.

The difference between the measured TI using scheme 1 and scheme 2 gives the effect of the asymmetry in the TIC' channels. The difference between the TI measured using scheme 2 and scheme 3 gives the effect of the asymmetry in the splitter' channels. Finally, the difference between the measured TI using scheme 1 and scheme 3 gives the asymmetry in the cables. Figure 6 shows the recorded measurements for the three counters using a triggering at both the positive and negative slopes. It is clear that the effect of the asymmetry in the splitter' channel is almost zero (the lowest two curves $(\mathbf{\square}, *)$ in Fig. 6). Also the detected asymmetry effect in both the TIC's channels and the cables is in the picosecond range (very small).

Note: All the asymmetry effects for TIC\# 2 are shifted slightly upward by about 5 ps. This can be explained by an internal offset in the trigger level adjustment of this counter.

\section{How to cancel out the effect of the internal error of the TIC}

To cancel out the effect of the precisely estimated internal error of the TIC, follow the following procedure:

(1) Before starting the TI measurement, use the schematic shown in Figure 3 (with $\Delta \ell=0$ ) in estimating the actual internal error of the counter using the same signal that is used in measuring the TI. Use the same measurement parameters that are used in measuring the TI. For example, use the same state for Averaging, Synchronization, Triggering Slope, and Triggering Level.

(2) Measure the TI.

(3) Subtract the estimated internal error from the measured TI.
By that way, we can precisely estimate the actual internal error of the TIC with the correct sign and cancel its effect.

As an application for this procedure, a $1 \mu \mathrm{s}$ standard TI was measured using SR 620 TIC (TIC\#1). The schematic shown in Figure 3 was used in measuring this TI with $\Delta \ell=8.4 \mathrm{~ns}$. The source of this $1 \mu \mathrm{s}$ TI was the complete cycle of a $1 \mathrm{MHz}$ high slew rate pulsed signal coming from a Hydrogen Maser frequency source. This signal is the same that was used in determining the internal error of the counter. The two channels of the counter were adjusted to the same triggering slope. Two cables of different length were used to make one channel starts at a certain rising edge while the second channel stops at the next rising edge. The measurement parameters are:

- Averaging of $1000(N=1000)$.

- Synchronized gate (external gating signal that was derived from the counter's timebase).

- The peak-to-peak of the $1 \mathrm{MHz}$ after the splitter is $1 \mathrm{~V}$ $(0.5 \mathrm{~V}:-0.5 \mathrm{~V})$

- An external HM based on time base of $5 \mathrm{MHz}$ was connected to the TIC.

To cancel out the effect of the estimated internal error, we have to subtract the value that was recorded using the same signal at the same trigger level and trigger slope from the measured TI. Also the difference in the cables length should be added to each measured TI.

Figure 7 shows that when we compensate for the estimated internal error of the TIC, we get more accurate results. For sure, the uncertainty is significantly decreased due to eliminating the effect of this major factor. For example, at $0 \mathrm{~V}$ trigger level, it is shown that the value of the measured TI is $999.696 \mathrm{~ns}$ while after subtracting the internal error the value is $1000.084 \mathrm{~ns}$.

\section{Conclusion}

Measuring the TI with the highest accuracy and least uncertainty is a main requirement in many applications that are running in the time and frequency laboratories. The internal error in the TIC used in the measurement is one of the significant factors that degrade the accuracy and measurement uncertainty. It was a common practice in the time and frequency labs to use the range given by the manufacturer in the uncertainty budget for this type of error. In general, the range given by the manufacturer is not wrong but is not accurate. In this paper a technique was proposed for estimating accurately the internal error of the counter. It was proved that this error depends on the shape of the signal being measured, the trigger level, and the trigger slope. Knowing precisely the value of this error enables us from completely eliminating its effect and enhancing the accuracy and reducing the measurement uncertainty of TI.

Acknowledgements. All the hardware equipments and signals that were used in the measurements are owned by the Physikalisch -Technischen Bundesanstalt (PTB) and used in 


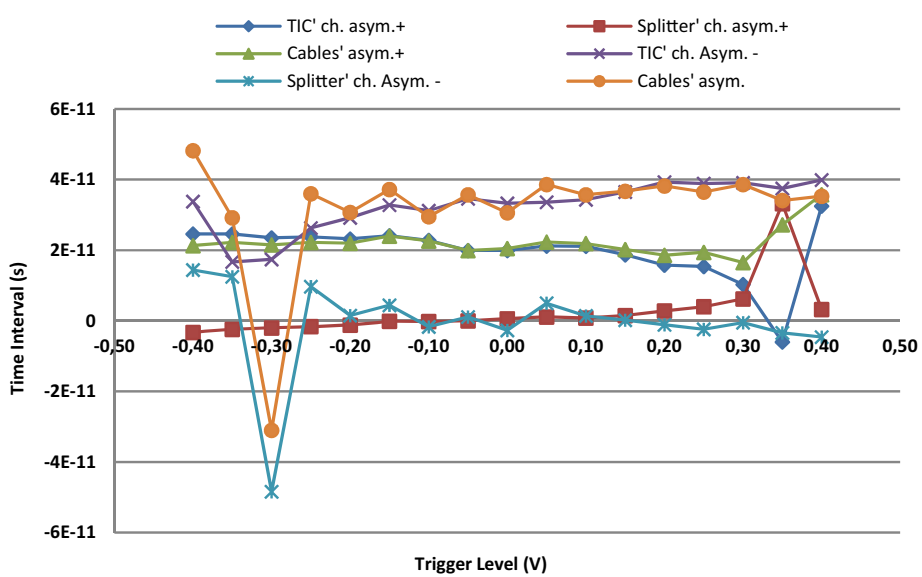

(a): The measured asymmetry in the TIC\# 1

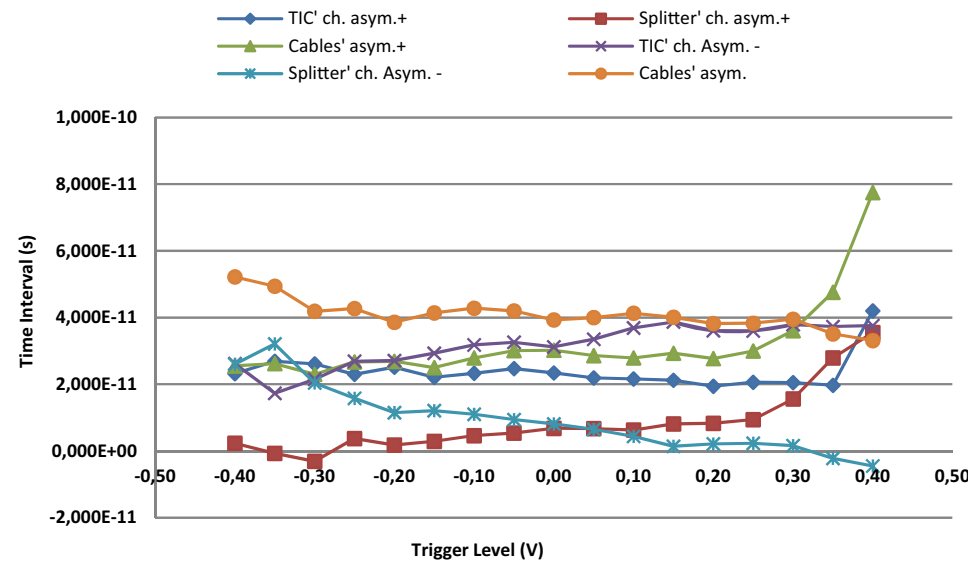

(b): The measured asymmetry in the TIC\# 2

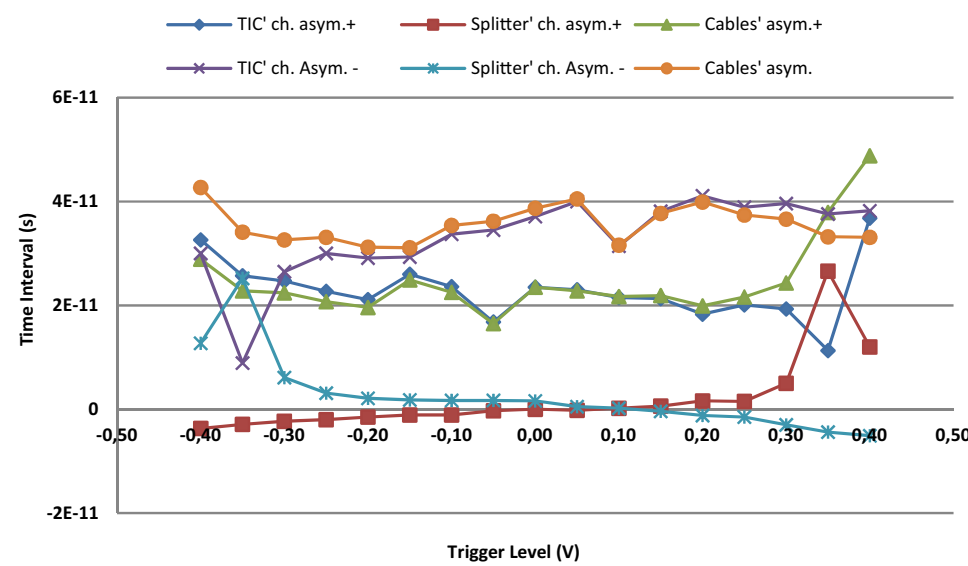

(c): The measured asymmetry in the TIC\# 3

Fig. 6. The measured asymmetry in the TIC' channels, the splitter, and the cables. TIC' ch. Asym.+: asymmetry in TIC' channels using positive slope triggering. Splitter' ch. Asym.t: asymmetry in splitter' channels using positive slope triggering. Cables' Asym.+: asymmetry in cables using positive slope triggering. TIC' ch. Asym.-: asymmetry in TIC' channels using negative slope triggering. Splitter' ch. Asym.-: asymmetry in splitter' channels using negative slope triggering. Cables' Asym.-: asymmetry in cables using negative slope triggering. 


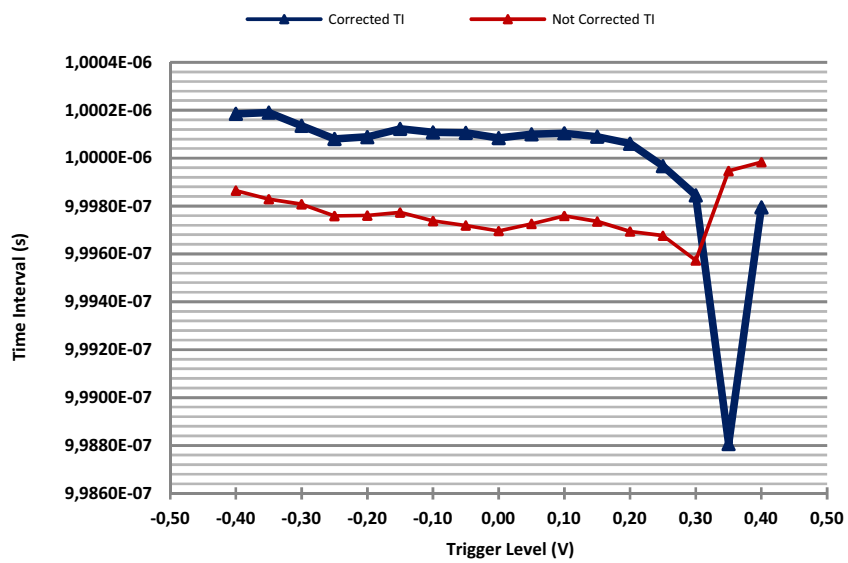

Fig. 7. Measuring a TI of $1 \mu$ s one time without removing the effect of the internal error and other time after removing its effect.

the measurements during my stay at PTB. So the author is grateful for PTB.

\section{References}

1. http://www . euramet.org/fileadmin/docs/projects/ 828_TIME_Final.pdf

2. http://www.euramet.org/index.php?id=tc-projects\&no_ cache $=1 \&$ tx_neurametctcp_projects $\% 5$ Bproject $\% 5 \mathrm{D}=$ 1064\&tx_nneurametctcp_projects $\% 5$ Baction $\% 5 \mathrm{D}=$ show \& tx_nneurametctcp_projects $\% 5$ Bcontroller $\% 5 \mathrm{D}=$ Project\& cHash $=5$ a485e5f4398a3ab53fe03d296dccb93

3. G.G. Hamza, Investigation of the Optimum Trigger Level in Time Interval Measurement, MAPAN-Journal of Metrology Society of India 29, 255-260 (12/2014)

4. G.G. Hamza, A Study on the EURAMET Comparison of Time Interval Measurement, MAPAN-Journal of Metrology Society of India 29, 207-212 (2014)

5. H.-N. Teodorescu, M. Hulea, Improving time measurement precision in embedded systems with a hybrid measuring method, in Proc. of the 6th IEEE International Conference on Intelligent Data Acquisition and Advanced Computing Systems: Technology and Applications, IDAACS'2011, 1, art. No. 6072711 (2011), pp. 59-64

6. M.L. Simpson, C.L. Britton, A.L. Wintenberg, G.R. Young, An integrated CMOS time interval measurement system with subnanosecond resolution for the WA-98 calorimeter, IEEE J. St. Circ. 32, 198-205 (1997)

7. B.K. Swann, B.J. Blalock, L.G. Clonts, D.M. Binkley, J.M. Rochelle, E. Breeding, K.M. Baldwin, A 100-ps timeresolution CMOS time-to-digital converter for positron emission tomography imaging applications, IEEE J. St. Circ. 39, 1839-1852 (2004)

8. http://kcdb.bipm.org/AppendixC/country_list.asp? type $=\mathrm{TF}$

9. Xiangwei Zhu, Shaowei Yong; Zhaowen Zhuang, A novel method with Ps accuracy for time interval measurement, in Proc. of the Frequency Control Symposium, 2007 Joint with the 21st European Frequency and Time Forum, IEEE International, pp. 848-853

10. Minshuang Huang, Junfen Huang, Yinqi Feng, High precision time-interval measurement method based on pseudonanuniform sampling, Advances in Automation and Robotics, Vol. 1, Lecture Notes in Electrical Engineering 122, 283-291 (2012)

11. Mircea Gh. Hulea, Horia-Nicolai L. Teodorescu, Method and system for time intervals measurement, Recent Patents on Electrical \& Electronic Engineering 5, 231-237 (2012)

12. J. Kalisz, Review of methods for time interval measurements with picosecond resolution, Metrologia 41, 17-32 (2004)

13. Better than 100 ps accuracy in HP 5370B time interval measurements through bias error reduction, 5370B Universal Time Interval Counter, Product Note 5370B-2, HEWLETT PACKARD

14. Sources of error in time interval measurements, Application note, Fluke

15. More hints for making better frequency counter measurements, Application note, Agilent

16. Understanding frequency counter specifications, Application Note 200-4, HEWLETT PACKARD

17. A. Bauch, S. Weyers, D. Piester, E. Staliuniene, W. Yang, Generation of UTC (PTB) as a fountain-clock based time scale (Metrologia, 2012)

18. P. Gibbs, Comparison of a single SR620 timer against a variety of timers from the Eurolas network, in Proc. of 13th International Workshop on Laser Ranging, Washington $D C(2002)$

19. P. Gibbs, A.T. Sinclair, Investigation of a small rangedependent bias of two SR620 Time Interval counters, in Proc. of 9th International Workshop on Laser Ranging Instrumentation (1994), pp. 274-276 